\title{
Partial hepatectomy hemodynamics changes: Experimental data explained by closed-loop lumped modeling.*
}

\author{
Chloe Audebert ${ }^{\mathrm{a}, \mathrm{b}}$, Mohamed Bekheit ${ }^{\mathrm{c}}$, Petru Bucur ${ }^{\mathrm{c}, \mathrm{d}}$, Eric Vibert ${ }^{\mathrm{c}, \mathrm{e}}$, Irene E. \\ Vignon-Clementel ${ }^{\mathrm{a}, \mathrm{b}, *}$ \\ ${ }^{a}$ Inria Paris, France \\ ${ }^{b}$ Sorbonne Universités UPMC Univ Paris 6, Laboratoire Jacques-Louis Lions, France \\ ${ }^{c}$ Inserm Unité 1193, Villejuif, France \\ ${ }^{d}$ CHRU, hôpitaux de Tours, Chirurgie Hépato-biliaire et Pancréatique, Transplantation Hépatique, Tours, France \\ ${ }^{e}$ AP-HP, Hôpital Paul Brousse, Centre Hépato-Biliaire, Villejuif, France
}

\begin{abstract}
The liver function may be degraded after partial liver ablation surgery. Adverse liver hemodynamics have been shown to be associated to liver failure. The link between these hemodynamics changes and ablation size is however poorly understood. This article proposes to explain with a closed-loop lumped model the hemodynamics changes observed during twelve surgeries in pigs. The portal venous tree is modeled with a pressure-dependent variable resistor. The variables measured, before liver ablation, are used to tune the model parameters. Then, the liver partial ablation is simulated with the model and the simulated pressures and flows are compared with post-operative measurements. Fluid infusion and blood losses occur during the surgery. The closed-loop model presented accounts for these blood volume changes. Moreover, the impact of blood volume changes and the liver lobe mass estimations on the simulated variables is studied. The typical increase of portal pressure, increase of liver pressure loss, slight decrease of portal flow and major decrease in arterial flow are quantitatively captured by the model for a $75 \%$ hepatectomy. It appears that the $75 \%$ decrease in hepatic arterial flow can be explained by the resistance increase induced by the surgery, and that no hepatic arterial buffer response (HABR) mechanism is needed to account for this change. The different post-operative states, observed in experiments, are reproduced with the proposed model. Thus, an explanation for inter-subjects post-operative variability is proposed. The
\end{abstract}

\footnotetext{
${ }^{\text {th }}$ A final version of this manuscript can be found in Journal of Biomechanics (2016)

* Corresponding Author

Email address: irene.vignon-clementel@inria.fr (Irene E. Vignon-Clementel)
} 
presented framework can easily be adapted to other species circulations and to different pathologies for clinical hepatic applications.

Keywords: Closed-loop lumped model; Hemodynamics; Surgery simulation; Hepatectomy; Validation; Swine.

\section{Introduction}

Major liver resection (partial hepatectomy) is being performed to treat liver lesions or for adultto-adult living donor liver transplantation. Due to liver regeneration, during the post-operative period of a few months, the patient re-gains a normal liver mass. However, sometimes liver function is poorly recovered and post-operative liver failure may occur.

Liver hemodynamics is modified by the surgery, which increases the resistance to flow of the organ. To understand it is not easy, partly because the liver is perfused by both arterial and venous blood. Although high portal pressure (Allard et al., 2013), high portal flow (Iida et al., 2007; Vasavada et al., 2014), and high hepatic venous pressure gradient (Sainz-Barriga et al., 2011) are associated with post-surgery liver failure, the link between resected volume and hemodynamics changes remains unclear. Since the liver receives around $25 \%$ of the cardiac output, hepatectomy may impact the whole blood circulation. Thus the present work aims to develop a mathematical model to explain the various hemodynamics changes observed in experimental surgeries of twelve pigs. Pig is considered a good animal model for liver.

The proposed model is constructed to satisfy the following requirements. First, the equations must be numerically fast to solve, to explore a diversity of hypotheses with all the pigs data. Second, the number of parameters must remain small enough so that calibration is tractable. Finally, the whole blood circulation must be taken into account, and hepatectomy dynamically modeled. Consequently, a closed-loop lumped model (also called 0D model), taking into account the liver and groups of organs, is presented.

Different groups have worked on liver hemodynamics modeling, at different liver scales and for various applications. Liver lobule porous models have been proposed, to model glucose transport 
and metabolism (Ricken et al., 2015), to study the influence of a septum and tissue permeability (Debbaut et al., 2014b), including in cirrhosis (Peeters et al., 2015) or to simulate the impact of deformation on pressure-flow relation (Bonfiglio et al., 2010). At the organ scale, liver $\pi$ lumped models for multiple vascular generations have been used to study the hypothermic machine perfusion (Van Der Plaats et al., 2004; Debbaut et al., 2014a). A lumped model of the splanchnic and liver circulation has been proposed to illustrate the link between hepatic venous pressure increase, vessel contractility and liver interstitial fluid (Chu and Reddy, 1992). Models have been developed on transport and diffusion of a compound in the liver, including whole-body pharmacokinetics models (Schwen et al., 2015) or to study tumor detection with Magnetic Resonance Images (Bezy-Wendling et al., 2007). Convection is based on resistive models of the different generations of arterial and venous trees. In (Lukeš et al., 2014), the flow in liver arterial and venous trees is modeled for the first generations with Bernouilli equation, while a porous media models the flow in the smallest vessels. The trees geometry is based on CT-scans. Hepatic artery flow 3D CFD simulations for rigid and flexible walls have been performed in (Childress and Kleinstreuer, 2014) to study direct drug-targeting. Liver models have also been developed to study the impact of liver surgery. Flow behavior for different $\mathrm{H}$-Graft diameters has been studied with a resistive model and compared to clinical observations in (Rypins et al., 1987). A 3D CFD simulation has been performed in the portal vein before and after right lobe hepatectomy in (Ho et al., 2012). The surgery was simulated by changing the geometry. Similarly, for a two-lobe liver lumped model, driving conditions were kept unchanged before and after hepatectomy. Various resection sizes and two different surgical techniques have been simulated using a resistance model, based on cast reconstruction, of rat liver vasculature (Debbaut et al., 2012). Most of these works thus do not consider the dynamics induced by the surgery or the interaction with the rest of the circulation.

The present work proposes to model liver partial ablation dynamically, with a closed-loop 0D model of the cardiovascular system and the liver. In (Audebert et al., 2016), we have proposed a numerical scheme for 1D hemodynamics models, and explored in a generic 1D-0D pig model the hepatic artery waveforms, to understand the experimental changes observed during hepatectomy. Here, the impact of the surgery on the liver and on the whole body hemodynamics is identified. Moreover 
the consequences of blood loss and infusion are studied. The simulations, done for twelve pigs, are quantitatively compared to experimental measurements from $75 \%$ pig hepatectomy. Prediction of hemodynamics changes relies partly on liver lobe masses. Thus, several options are tested. The paper is organized in the following manner. Section 2 presents the available experimental measurements, the cardiovascular and liver models and their parametrization. Section 3 shows partial hepatectomy simulation results and comparison with measurements. Section 4 discusses model capabilities.

\section{Methods}

\subsection{Liver surgery - experimental measurements}

Hepatectomies are performed on several pigs to study the hemodynamics impacts. Approval of the committee of ethics of animal research, ministry of higher education and scientific research and ministry of agriculture and fishing was obtained. The pig liver is composed of five lobes, usually considered as three main lobes (Court et al., 2003): left lobe, median lobe (subdivided in left medial and right medial lobes) and right lobe (subdivided in right lateral and caudate lobes). The median and left lobes are resected. Since the median lobe is around twice the size of left and right lobes, around $75 \%$ hepatectomy is performed.

During surgery, several measurements are continuously recorded. Three pressures and three flows are the basis of parameter tuning and model validation. These measurements are averaged over 20 seconds during a stable state of the surgery. Pre-resection and post-resection (immediately after surgical clamping) states are considered. The carotid artery $(\mathrm{CA})$, portal vein $(\mathrm{PV})$ and central venous $(\mathrm{CV})$ pressures are measured. The later is a surrogate for the hepatic vein (v) pressure. The flows are recorded in the aorta above the celiac trunk (celiac aorta), the hepatic artery (HA) and the portal vein. Cardiac output $(\mathrm{CO})$ is estimated assuming celiac aorta flow is around $60 \%$ of $\mathrm{CO}$ (Lantz et al., 1981) (assuming humans and pigs flow distributions are similar (Swindle et al., 2012)). Heart rate is computed from the CA pressure measurement.

Before and after the surgery a CT-scan is performed with a Siemens Somatom AS definition 128 machine. Image acquisitions are done before, 15, 35, 55 and 75 seconds after injection of $75 \mathrm{ml}$ of iohexol $350 \mathrm{mg} / \mathrm{ml}$ (Omnipaque, GE Healthcare) with a rate of $5 \mathrm{ml}$ per second. From the CT-scans liver volumes are estimated. After ablation, the removed liver is weighted; left and median lobe 


\begin{tabular}{|c|c|c|c|c|}
\hline Mass assumption & A1 & A2 & A3 & A4 \\
\hline Total liver mass & $\begin{array}{c}\text { estimate with } \\
\text { pre-op CT-scan }\end{array}$ & $\begin{array}{c}\text { estimate with } \\
\text { pre-op CT-scan }\end{array}$ & $\begin{array}{c}\text { sum of lobe } \\
\text { masses }\end{array}$ & $\begin{array}{c}\text { sum of lobe } \\
\text { masses }\end{array}$ \\
\hline Left lobe mass & $\begin{array}{c}1 / 3 \text { planned resected } \\
\text { mass (pre-op CT-scan) }\end{array}$ & $\begin{array}{c}\text { weight after } \\
\text { resection }\end{array}$ & $\begin{array}{c}\text { weight after } \\
\text { resection }\end{array}$ & $\begin{array}{c}\text { weight after } \\
\text { resection }\end{array}$ \\
\hline Right lobe mass & $\begin{array}{c}\text { planned remaining } \\
\text { mass (pre-op CT-scan) }\end{array}$ & $\begin{array}{c}\text { total mass minus } \\
\text { left and median lobe masses }\end{array}$ & $\begin{array}{c}\text { equal to left } \\
\text { lobe mass }\end{array}$ & $\begin{array}{c}\text { estimate with } \\
\text { post-op CT-scan }\end{array}$ \\
\hline Median lobe mass & $\begin{array}{c}2 / 3 \text { planned resected } \\
\text { mass (pre-op CT-scan) }\end{array}$ & $\begin{array}{c}\text { weight after } \\
\text { resection }\end{array}$ & $\begin{array}{c}\text { weight after } \\
\text { resection }\end{array}$ & $\begin{array}{c}\text { weight after } \\
\text { resection }\end{array}$ \\
\hline
\end{tabular}

Table 1: The different mass assumptions description of the total liver, left lobe, right lobe and median lobe. Their degree of certainty increases, and conversely their degree of predictability decreases from A1 to A4: preop calculation, peri-op calculation possible, post-op calculation.

masses are then assessed. To estimate the liver masses, four different assumptions are made as described in Table 1, with varying predictive capabilities.

\subsection{OD closed-loop model}

A 0D hemodynamics model of the entire cardiovascular system (Liang and Liu, 2005; Segers et al., 2003) is coupled to a new model of liver that is structured by lobes. The model aims to represent hepatectomy, i.e. the resection but also other related phenomena. Hence, only the involved organs are included, resulting in five blocks (figure 1).

Lungs $(i=L)$, digestive organs $(i=D O)$ and other organs $(i=O O)$ are represented by threeelement Windkessel models:

$$
\left\{\begin{array}{l}
C^{i} \frac{d P_{p}^{i}}{d t}=Q_{a}^{i}-Q_{v}^{i} \\
R_{p}^{i} Q_{a}^{i}=P_{a}^{i}-P_{p}^{i} \\
R_{d}^{i} Q_{v}^{i}=P_{p}^{i}-P_{v}^{i}
\end{array}\right.
$$

where for block $i Q_{a}^{i}$ and $Q_{v}^{i}$ are arterial and venous flows, $P_{a}^{i}, P_{p}^{i}$ and $P_{v}^{i}$ are arterial, proximal and venous pressures, $R_{p}^{i}, R_{d}^{i}$ and $C^{i}$ are proximal and distal resistances and capacitance (figure 1).

Heart model. The heart model is based on (Suga and Sagawa, 1974; Pennati et al., 1997; Liang et al., 2009; Blanco and Feijóo, 2013). To obtain smooth, yet sharp, transitions between open and closed valves, logistic functions are used for valves (Audebert et al., 2016). 
Liver model. The liver tissue is perfused with venous blood through PV and arterial blood through HA and drained by the hepatic vein. The liver main lobes are represented by three blocks in parallel (figure 1), related to the left heart (arterial input), digestive organs (venous input) and right heart (venous output) compartments. Within each lobe, the HA tree is represented by a single resistance. The PV tree is modeled by a non-linear resistance to represent pressure $(P)$ dependent resistance $(R)$, the subscript 0 labelling before resection:

$$
P-P_{0}-K\left(\left(\frac{R_{0}}{R}\right)^{5}-\left(\frac{R_{0}}{R}\right)^{-0.75}\right)=0
$$

This equation is derived from a vein tube law relating pressure and area (Shapiro, 1977; Pedley et al., 1996): $P-P_{0}=K\left(\left(A / A_{0}\right)^{10}-\left(A / A_{0}\right)^{-1.5}\right)$. Then, the area and the resistance are related assuming Poiseuille flow. Finally, liver tissue and hepatic vein trees are modeled with a capacitance and a resistance (Bennett and Rothe, 1981).

The resistances and capacitance in a lobe are assumed, as a first approximation, proportional to lobe mass inverse and lobe mass respectively. Thus, for each lobe:

$$
\left\{\begin{aligned}
P_{a}-P_{t, i} & =R_{h a} \frac{M}{M_{i}} Q_{a, i} \\
P_{p v}-P_{t, i} & =R_{p v}\left(P_{p v}\right) \frac{M}{M_{i}} Q_{p v, i} \\
P_{t, i}-P_{v} & =R_{l} \frac{M}{M_{i}} Q_{v, i} \\
C_{l} M_{i} \frac{d P_{t}}{d t} & =Q_{a, i}+Q_{p v, i}-Q_{v, i}
\end{aligned}\right.
$$

where $P_{a}, P_{p v}, P_{t, i}$ and $P_{v}$ are respectively the pressure for artery, portal vein, $i$ th lobe tissue and venous pressure. $Q_{a, i}, Q_{p v, i}$ and $Q_{v, i}$ are $\mathrm{HA}, \mathrm{PV}$ and hepatic venous flows in lobe $i$ respectively. $M$ and $M_{i}$ are the liver and lobe $i$ mass estimations. $R_{h a}, R_{p v}, R_{l}$ are resistances for HA tree, PV tree, and liver tissue and hepatic venous tree. $C_{l}$ is the liver tissue capacitance per liver mass, the only liver non pig-specific parameter. 
Partial hepatectomy simulation. To model median and left lobes resection, the corresponding HA and PV resistances are multiplied by a function dependent on $T_{75}$, the resection time:

$$
r(t)=\left\{\begin{array}{l}
1 \text { if } t<T_{75} \\
\exp \left(5\left(t-T_{75}\right)\right) \text { otherwise }
\end{array}\right.
$$

Infusion or bleeding modeling. During surgery, blood and lymph losses and evaporation due to openabdomen occur. Anesthetists thus infuse fluid. Consequently blood volume is not constant. In the model, systemic venous flow is thus changed dynamically by adding or removing flow to represent bleeding or infusion (Figure 1):

$$
Q_{i, b}(t)=\frac{V_{i / b}}{D_{i / b}(1+\exp (-0.1(\Delta t-0.01)))} \quad \text { with } \Delta t= \begin{cases}1 & T_{i / b} \leq t \leq T_{i / b}+D_{i / b} \\ 0 & \text { otherwise }\end{cases}
$$

Where $T_{i / b}$ and $D_{i / b}$ are the time and duration of the infusion or bleeding and $V_{i / b}$ is the added or removed volume.

Numerical resolution. The model leads to a system of nonlinear differential-algebraic equations, solved with the IDA package from SUNDIALS (Serban et al., 2015). A Backward Differentiation Formula is used for time integration, and a Newton method for the resulting nonlinear system.

Statistic tests. A test of equivalence compares the model outputs and the measurements. The equivalence test null hypothesis is the dissimilarity of the two populations. Thus, the test assumes the populations are different and uses the data to prove otherwise (Robinson et al., 2005). A two one-sided $t$-test (TOST) is used, with the R function TOST in the "equivalence" package (R Development Core Team, 2008; Robinson, 2016). For the equivalence test, a region of indifference has to be defined; here a $10 \%$ relative error is chosen. If the region of indifference is contained in the confidence interval, then the two populations are deemed significantly similar. If not, the null hypothesis is not rejected (Robinson and Froese, 2004). 

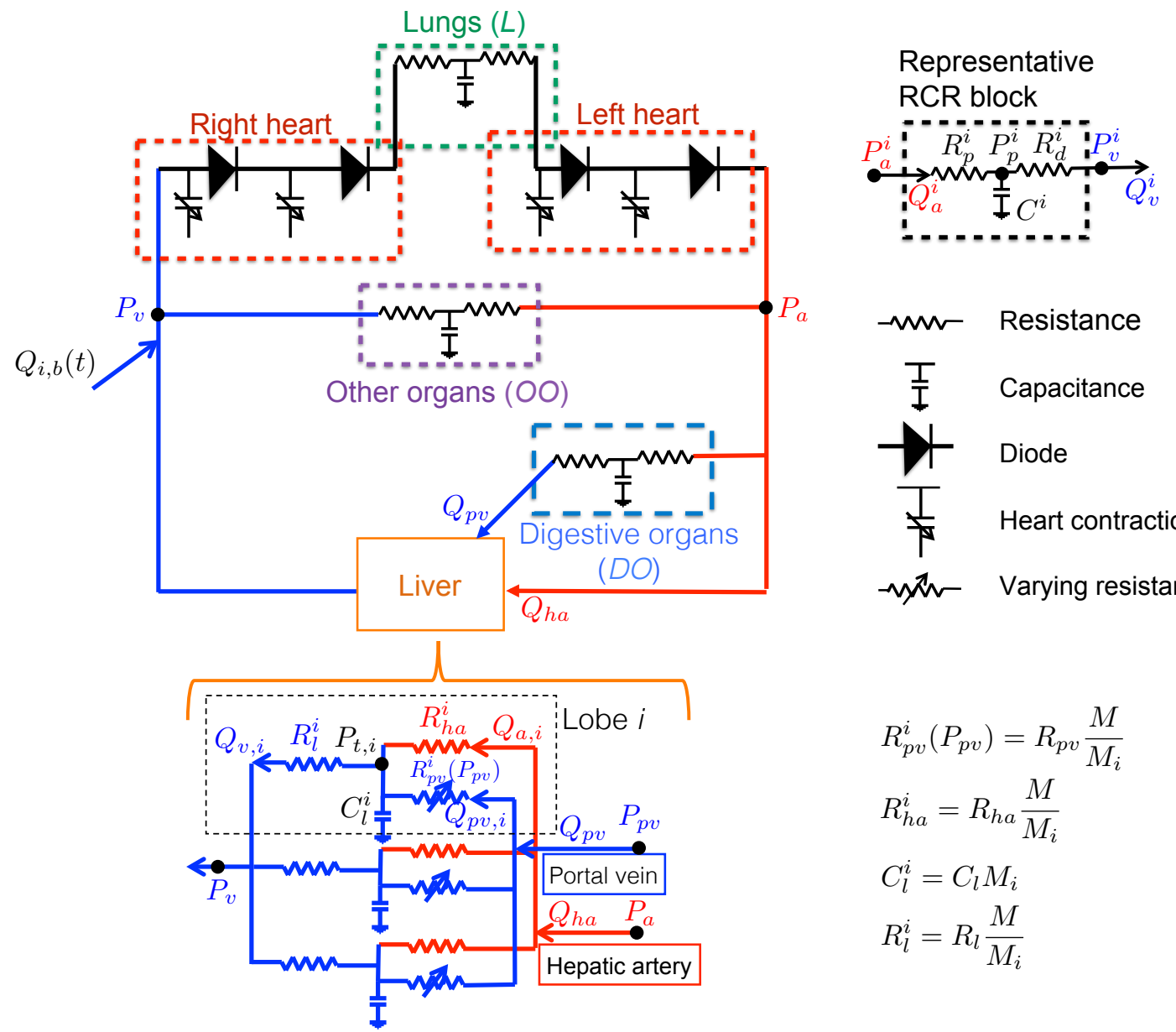

Resistance

I $\quad$ Capacitance

$\checkmark$ Diode

$\frac{1}{1}$ Heart contraction

-yMh Varying resistance

$R_{p v}^{i}\left(P_{p v}\right)=R_{p v} \frac{M}{M_{i}}$

$R_{h a}^{i}=R_{h a} \frac{M}{M_{i}}$

$C_{l}^{i}=C_{l} M_{i}$

$R_{l}^{i}=R_{l} \frac{M}{M_{i}}$

Figure 1: Schematic representation of the 0D closed-loop cardiovascular and liver blood circulations. RCR block and liver lobe $i$ parameters are shown. $Q_{i, b}$ is the infused or removed flow to account for blood volume changes. 


\subsection{Parameter tuning procedure}

Based on the available cardiac-cycle-averaged measurements and literature data, model parameters are tuned to obtain similar pressures and flows to the measured ones before resection.

Systemic circulation. CA and CV pressure measurements are used as target arterial $\left(P_{a}\right)$ and venous $\left(P_{v}\right)$ pressures in the model. Combined with $C O$, the systemic equivalent resistance is computed: $R_{e q}=\left(P_{a}-P_{v}\right) / C O$. Similarly, the RCR total resistance for DO is computed with measured $P_{a}$, $P_{p v}$ and $Q_{p v}$. The liver tissue pressure $\left(P_{t}\right)$ is estimated assuming that the pressure drop between $\mathrm{PV}$ and liver tissue is $80 \%$ of the pressure drop between $\mathrm{PV}$ and hepatic vein $\left(P_{p v}-P_{v}\right)$ (Debbaut et al., 2012). Combined with the $P_{a}$ and $Q_{h a}$ measurements, the HA tree resistance is computed:

$$
R_{h a}=\frac{P_{a}-P_{t}}{Q_{h a}}
$$

Similarly, the liver tissue resistance and the initial portal resistance $\left(R_{0}\right.$ in equation $\left.(2)\right)$ are computed:

$$
R_{l}=\frac{P_{t}-P_{v}}{Q_{h a}+Q_{p v}} ; \quad R_{p v}=\frac{P_{p v}-P_{t}}{Q_{p v}}
$$

The $O O$ total resistance is then computed: $R_{O O}=1 /\left(1 / R_{e q}-1 / R_{h a}-1 / R_{D O}\right)$. In the RCR models, the proximal resistance is assumed to carry $10 \%$ and $5 \%$ of the total resistance, for the $D O$ and OO blocks respectively, within ranges in (Raines et al., 1974; Vignon-Clementel et al., 2010; LaDisa et al., 2011).

Capacitances are fixed (same for all pigs). Other organs capacitance: $C^{O O}=7.3610^{-4} \mathrm{~cm}^{5} / \mathrm{dyn}^{O}$ (within ranges in (Segers et al., 2000) ; digestive organs capacitance (manually tuned): $C^{D O}=$ $410^{-4} \mathrm{~cm}^{5} /$ dyn and liver tissue capacitance per mass: $C_{l}=1.510^{-5} \mathrm{~cm}^{5} / \mathrm{dyn} / \mathrm{g}$ (Bennett and Rothe, 1981).

Heart and lung parameters. The amplitude and baseline of heart contraction functions are tuned, for each pig, to obtain arterial and venous average pressures measured before resection. The heart contraction times and cardiac cycle are based on the measured heart rate (Table 2). Lung parameters 


\begin{tabular}{|c|c|c|}
\hline Notation & Detail & Value in second \\
\hline$T_{c c}$ & Cardiac cycle & $60 \mathrm{~s} /$ heart rate $(\mathrm{bpm})$ \\
$T_{v c}$ & Ventricular contraction duration & $0.34 T_{c c}$ \\
$T_{v r}$ & Ventricular relaxation duration & $0.15 T_{c c}$ \\
$T_{a c}$ & Atrium contraction duration & $0.17 T_{c c}$ \\
$T_{a r}$ & Atrium relaxation duration & $0.17 T_{c c}$ \\
$t_{a c}$ & Time atrium begins to contract & $0.8 T_{c c}$ \\
$t_{a r}$ & Time atrium begins to relax & $0.97 T_{c c}$ \\
\hline
\end{tabular}

Table 2: Durations and times for contraction and relaxation of the different cardiac chambers. These parameters are the same for the left and right hearts.

are based on (Blanco and Feijóo, 2013): $R_{p}^{L}=53.33$ dyn.s $/ \mathrm{cm}^{5} ; R_{d}^{L}=53.33$ dyn.s $/ \mathrm{cm}^{5}$ and $C^{L}=$ $0.05 \mathrm{~cm}^{5} / \mathrm{dyn}$.

\section{Results}

Hepatic surgeons are particularly interested in specific pressure and flow values hypothesized to be linked to liver failure. The variables of interest are: arterial pressure, PV pressure, the venous pressure drop $P_{p v}-P_{v}$ across the liver, PV flow and HA flow. Simulated and measured values are compared for these variables.

\subsection{Pre-resection stage}

The tuning procedure described above (2.3), based on pre-resection measurements, gives good agreement between pre-resection simulated and measured values. Figure 2 displays the simulated pressures and flows of interest against the measured ones in logarithmic scale. The dots in figure 2 are nicely aligned along the curve $y=x$ illustrating the good match between the results and measurements, for all pigs. Parameters are tuned for each animal, thus the inter-animal variability is well captured. Standard deviations for measured and simulated variables are: $10.4 \mathrm{mmHg}$ for arterial pressure, $2.4 \mathrm{mmHg}$ for $\mathrm{PV}$ pressure, $3.1 \mathrm{mmHg}$ for the pressure drop, $0.06 \mathrm{~L} / \mathrm{min}$ and 0.18 $\mathrm{L} /$ min for $\mathrm{HA}$ and $\mathrm{PV}$ flow rates respectively.

\subsection{Liver partial resection simulation}

Impact of liver lobe mass assumptions. The post-resection simulated variables are impacted by the estimation of the liver lobe masses. Thus, the simulations are run, with the four different mass 


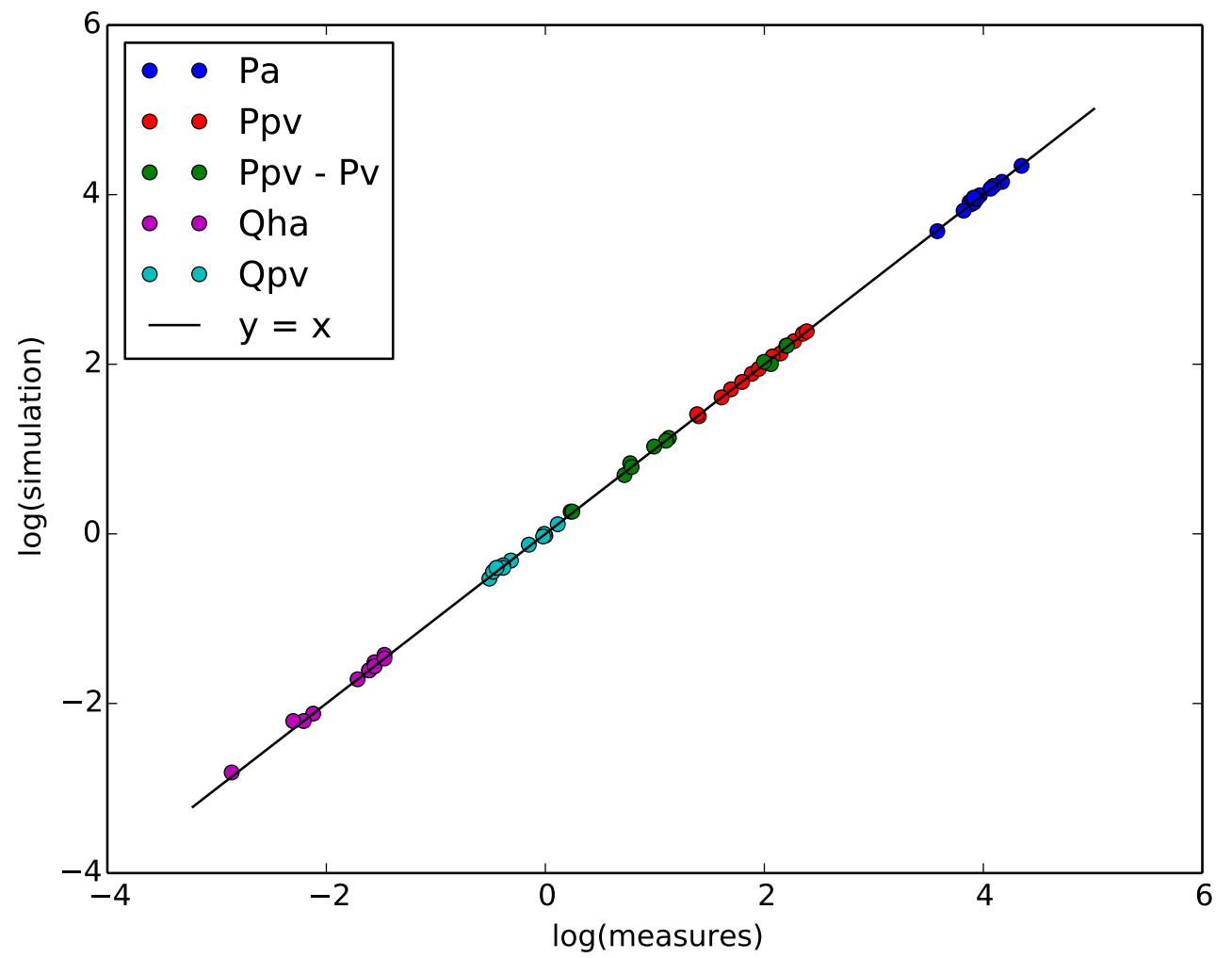

Figure 2: Pre-resection measurements vs simulation values in $\log / \log$ scale, for each variable (unique color) and for each animal (one dot). Pressures are in $\mathrm{mmHg}$ and flow rates are in $\mathrm{L} / \mathrm{min}$. 


\begin{tabular}{|ccccc|}
\hline Mass assumption & $\mathrm{A} 1$ & $\mathrm{~A} 2$ & $\mathrm{~A} 3$ & $\mathrm{~A} 4$ \\
\hline$P_{a}$ & $0.2(0.18)$ & $0.2(0.18)$ & $0.21(0.18)$ & $0.2(0.18)$ \\
$P_{p v}$ & $0.28(0.19)$ & $0.46(0.57)$ & $0.47(0.46)$ & $0.37(0.26)$ \\
$P_{p v}-P_{v}$ & $0.39(0.26)$ & $0.74(0.89)$ & $0.56(0.49)$ & $0.46(0.38)$ \\
$Q_{h a}$ & $0.63(0.59)$ & $0.65(0.75)$ & $0.38(0.27)$ & $0.27(0.27)$ \\
$Q_{p v}$ & $0.48(0.62)$ & $0.42(0.59)$ & $0.4(0.6)$ & $0.44(0.6)$ \\
\hline
\end{tabular}

Table 3: Mean and standard deviation (in parenthesis) of relative error $\mathcal{E}_{r e l}$ for all the 12 simulations, $\mathcal{E}_{\text {rel }}=$ $\left|X_{\text {simu }}-X_{m e s}\right| /\left|X_{m e s}\right|$ with $X_{\text {simu }}$ the simulated and $X_{m e s}$ the measured post-resection variable.

estimations (Table 1), for twelve different pigs and compared with measurements. Table 3 shows mean and standard deviation of the relative error for the different variables and mass assumptions. The arterial pressure and PV flow are almost not impacted by the lobe mass differences (at most $20 \%$ difference). The lobe mass estimations have a significant impact on PV pressure (at most $68 \%$ difference), the pressure drop (at most 90\% difference), and HA flow (140\% maximum difference). The last mass estimation gives the smallest relative errors (in average), thus this mass assumption is kept for the rest of the simulations. It is indeed the least predictive assumption but gives as expected the best simulation results.

Hepatectomy simulation. The simulation results averages for twelve pigs are compared to the measurements (Figure 3) before and after resection. After liver resection, on average, $45 \%$ increase of PV pressure and $98 \%$ increase in pressure drop are measured. A small decrease in arterial pressure of $12 \%$ is observed. Moreover, a large decrease in HA flow, $74 \%$, and a smaller decrease in PV flow, $30 \%$, are measured. In the model, in average, $66 \%$ increase for PV pressure and $110 \%$ increase for pressure drop are simulated. The fact that these pressures increase is coherent with the measurements, but these increases are overestimated. The arterial pressure decrease is only $3 \%$. The model underestimates the decrease of PV flow (5\%), but captures well the HA flow decrease of $75 \%$.

Taking into account changes in blood volume. During surgery, the total volume of blood in the circulation varies. Estimating its loss or gain is complex. However, pressures are strongly linked to the circulating blood volume. Thus, the changes observed in arterial pressure measurements are used to estimate the change in blood volume. The volume added or removed is chosen such that the simulated post-resection arterial pressure corresponds to the measurement. Therefore a decrease of $12 \%$ in arterial pressure is obtained with the model. 


\begin{tabular}{|cc|cc|}
\hline & Before resection & \multicolumn{2}{|c|}{ After resection } \\
& & Blood volume constant & Blood volume changes \\
\hline $\begin{array}{c}\text { p-value } \\
\text { result }\end{array}$ & $3.310^{-23}$ & 0.94 & 0.027 \\
& Reject & Not reject & Reject \\
\hline
\end{tabular}

Table 4: p-values for TOST test between the 12 simulated post-resection variables and measurements. The simulations include or not the changes in blood volume.

Figure 3 displays the simulated variables, taking into account the change in blood volume, averaged over twelve pigs. Pressures are largely impacted by changes in blood volume. The model agrees well with the measurements for PV pressure and pressure drop, with increases of $43 \%$ and $82 \%$ respectively. HA flow is still correctly simulated, with a $79 \%$ decrease, and PV flow is improved, with $23 \%$ decrease, however is still overestimated.

Similarity of measured and simulated populations. The model ability to reproduce the animal population variability is verified with a two one-sided $t$-test. The A4 mass assumption is used to perform the simulations. The test is performed with and without blood volume changes. For each variable of interest and each simulation the relative error is computed. The test is performed on the obtained relative error vectors. The results and p-values are given in Table 4. According to the test p-values the null hypothesis is rejected for the pre-resection simulation, with $10 \%$ relative error for indifference region. More precisely, the simulated variables and the measurements, before resection, are significantly similar. The null hypothesis is not rejected for post-resection simulation without blood loss changes, with $10 \%$ relative error for indifference region. Meaning, there is insufficient evidence to reject the null hypothesis. This result may occur, because the model output and the measurements really differ or because the sample size is too small to conclude. Finally, with a $10 \%$ relative error indifference region, post-resection simulation with blood volume changes and measurements are significantly similar. Taking into account blood volume changes knowing the change in arterial pressure, improves model outputs.

\section{Discussion and conclusion}

Measurements explained by modeling. The behavior of the measured pressures and flows during $75 \%$ hepatectomy are analyzed using the model. The observed HA flow decrease corresponds exactly to 


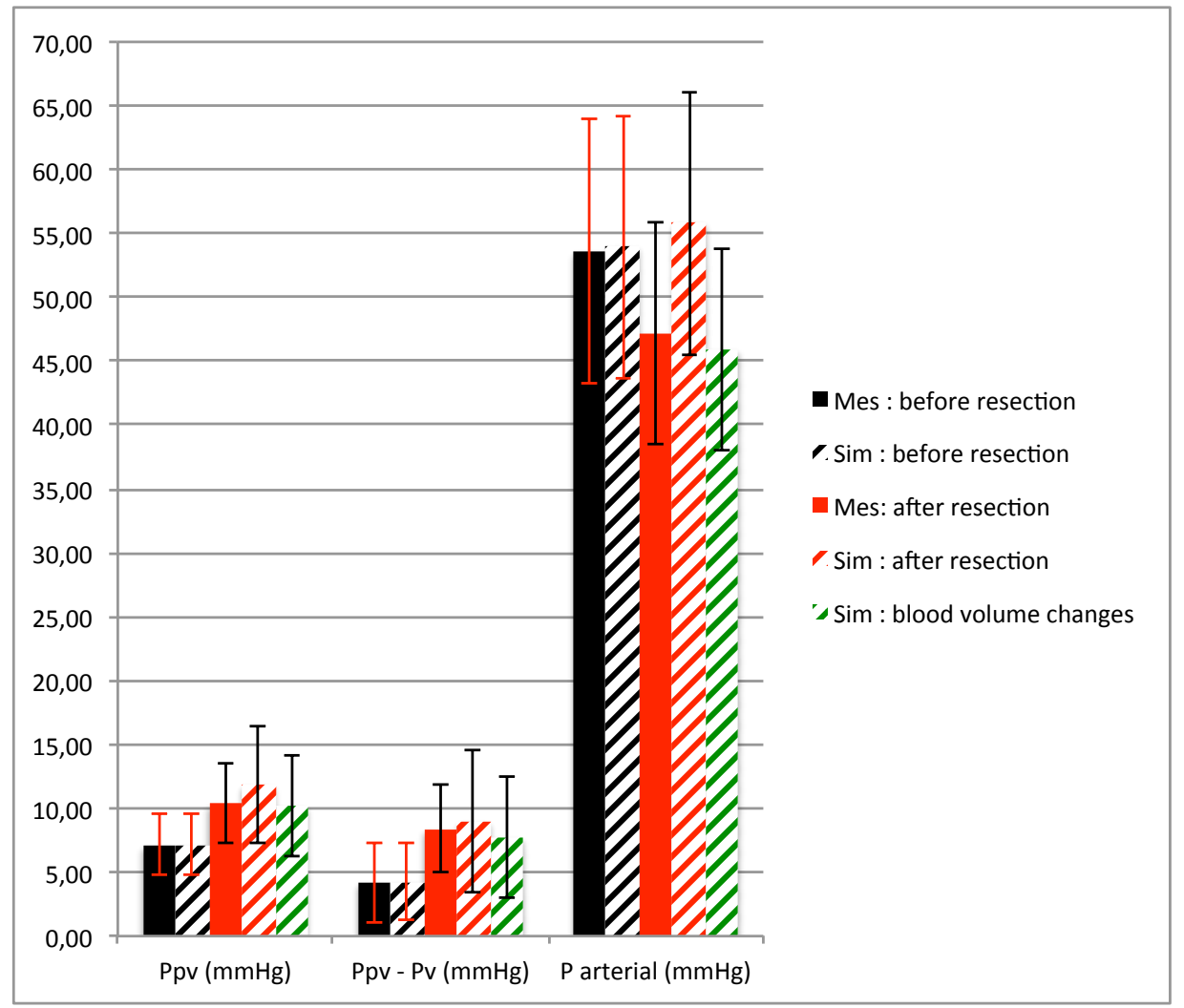

(a) Pressures

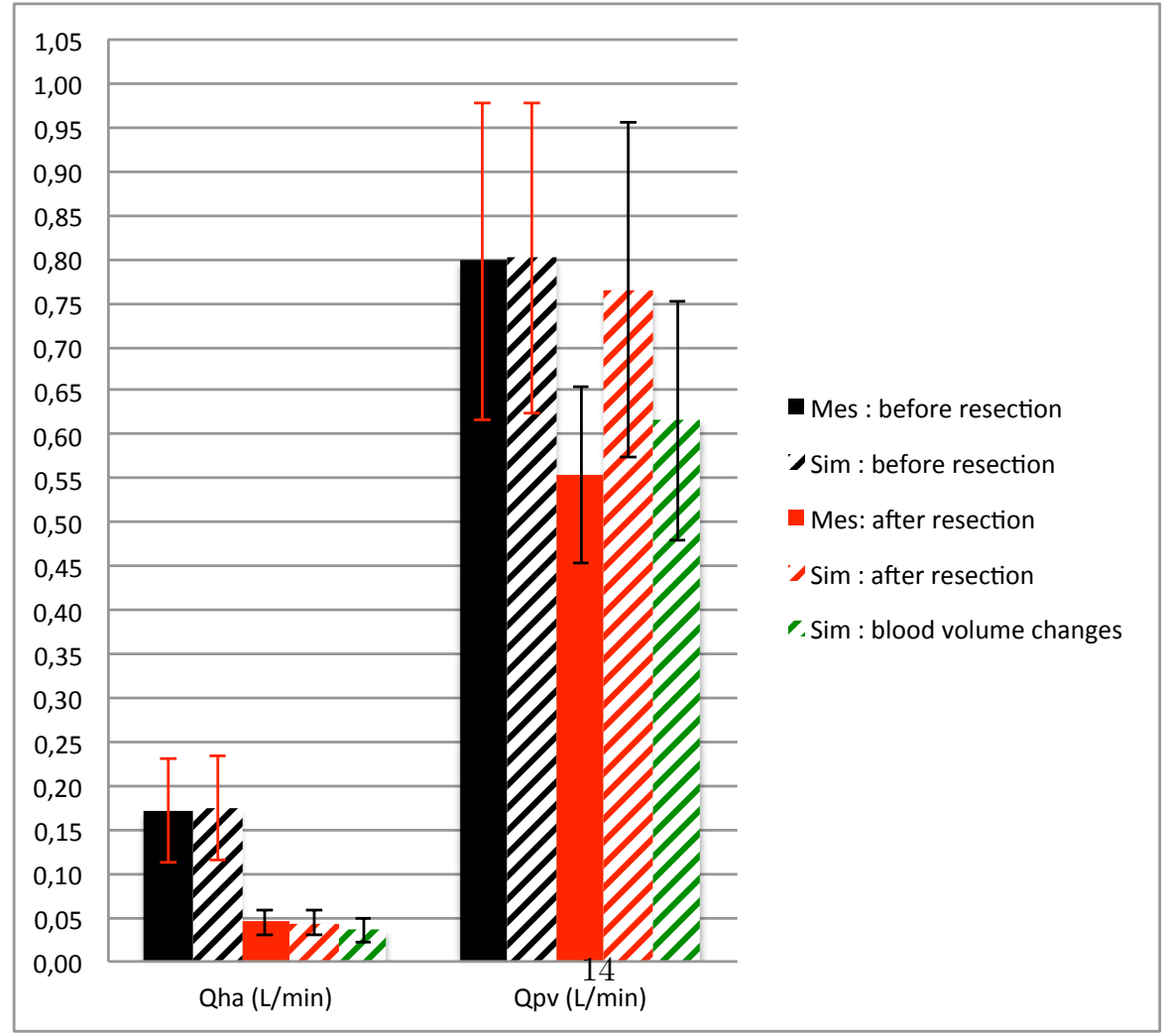

(b) Flows

Figure 3: Measurements (full) and simulations (dash) at different states of the surgery: pre-resection, post-resection. Simulations with (dashed green) and without (dashed red) blood volume changes are represented for the A4 mass assumption (a) pressures and (b) flows. 
the increase of the HA tree resistance due to the $75 \%$ liver resection, without HABR being needed. At leading order, the liver arterial system behaves as $\Delta P=R Q$, with $\Delta P$ the arterial pressure drop, which remains almost constant, $R$ the liver resistance and $\mathrm{Q}$ is the HA flow. The $75 \%$ liver resection induces the HA tree resistance increase of $75 \%$, thus explaining the decrease of HA flow. The arterial pressure is not impacted by the liver resection because the HA and liver resistances are small compared to the rest of the systemic circulation. However in average it decreases by $12 \%$ in the measurements. This decrease is a consequence of the blood loss, as proven with the model. PV flow measurements, in average, decrease by $30 \%$. The main decrease is due to blood (volume) loss. This is reinforced by the fact that animals with larger blood losses have a more important PV flow decrease. However the simulations, without blood loss, show that $75 \%$ liver resection accounts for a decrease of portal flow of around $5 \%$. The measured PV pressure and venous pressure drop increase by $45 \%$ and $98 \%$ respectively. The portal pressure increase is expected given the increase of PV tree and liver resistances due to resection. However it is compensated by three mechanisms: interaction with the rest of the circulation which causes PV flow to decrease, the lessen increase in $\mathrm{PV}$ tree resistance due to dilation and the general pressure decrease due to blood loss. Indeed, the simulations without blood volume change predict a $66 \%$ increase of portal pressure and adding the blood losses the increase is $43 \%$. If the venous pressure remains constant during surgery, the pressure drop would increase by $110 \%$ as simulated with the model. However, the measured CV pressure decreases by $33 \%$. Thus, the blood losses lead to all venous pressures decrease, and an increase in the pressure drop estimated as $82 \%$ in the simulations.

Sensitivity and variability. The liver lobe mass estimations impact the post-resection simulated variables due to the fact that the liver lobe resistances and capacitances depend on lobe masses. The mostly impacted variables are PV pressure, the pressure drop and HA flow. This is expected since arterial pressure and portal flow, are strongly dependent on heart and digestive organs parameters respectively. A better estimation of the liver lobe masses may improve post-resection simulated variables.

Moreover, several events happen during the surgery due to surgical acts, anesthetists interventions etc. Here, the model demonstrates that taking into account the change in blood volume improves the simulated post-resection prediction, knowing e.g. the change in arterial pressure measurements. 
In terms of variability, the simulated results are in good agreement with the measurements, both for pre-resection and post-resection with blood volume changes, according to the TOST tests. The tests also show that taking into account blood loss significantly improved the model outputs agreement with the measurements.

Pressure and flow changes due to hepatectomy without any volume change are also simulated with the model. Theses results may represent the state after the surgery, once the blood volume is back to the pre-resection volume. Under this assumption, the portal pressure and pressure drop, important for liver failure (Allard et al., 2013), may be underestimated with intraoperative measurements. Thus, for a $75 \%$ liver ablation, the model predicts an increase, in the following post-operative days of $110 \%$ instead of $82 \%$ for the pressure drop and $66 \%$ instead of $43 \%$ for portal pressure.

The simulation results for each animal are presented in Table 6. Among animals, various postresection behaviors are obtained with the simulations. For example, without change in blood volume, for animal iF03 PV flow is almost unchanged (decrease by $1.7 \%$ ) compared to iF12 for which it decreases by $13 \%$ (Table 6 ). The simulated blood volume change is adapted on an animal basis to the arterial pressure change. For animal iF02 a loss of $200 \mathrm{ml}$ is simulated compared to animal iF08 for which a volume infusion of $200 \mathrm{ml}$ is modeled (Table 6). Therefore, the model is able to simulate the different hemodynamics states that occur post-resection.

Future work. In summary, this work presents the first dynamics model of liver ablation. Its validation based on pig data is promising; the model is able to capture and explain the main features of hemodynamics changes due to the surgery as well as its variability among pigs, and may give insights about other states (e.g. day post-surgery) when measurements are difficult to take. HABR does not seem needed to explain the data. Future work will include the adaptation of the liver model to human liver anatomy. Moreover, in this work, several pressure and flow measurements were available at different stages of the surgery. The pre-resection measurements were used for parameter tuning. In patient surgeries less measurements are available. Furthermore, the model and measurements in this work are for a healthy (pig) liver. However the liver of the patients treated with partial hepatectomy is generally not healthy. For example, collateral circulations can appear (Pinzani and Vizzutti, 2005), and the liver resistance and capacitance parameters can change. Thus, future work is needed to integrate these considerations. Despite this, the framework in place can easily be adapted to 
include various measurements and different pathologies for clinical applications. Finally, the model can be a basis to compute how much to control portal flow in order to avoid liver-failure related hemodynamics ranges (Iida et al., 2007; Vasavada et al., 2014; Sainz-Barriga et al., 2011; Allard et al., 2013).

\section{Acknowledgments}

This material is based upon work supported by the French National Agency for Research ANR13-TECS-0006 iFLOW. The authors are grateful to the INRA Plateforme CIRE (Nouzilly, France) staff for their technical assistance in surgeries and imaging, and to Mylène Wartenberg for assistance in taking measurements.

\section{Conflict of interest}

None declared.

\section{References}

Allard, M.A., Adam, R., Bucur, P.O., Termos, S., Cunha, A.S., Bismuth, H., Castaing, D., Vibert, E., 2013. Posthepatectomy portal vein pressure predicts liver failure and mortality after major liver resection on noncirrhotic liver. Annals of surgery 258, 822-830.

Audebert, C., Bucur, P., Bekheit, M., Vibert, E., Vignon-Clementel, I.E., Gerbeau, J.F., 2016. Kinetic scheme for arterial and venous blood flow, and application to partial hepatectomy modeling. Computer Methods in Applied Mechanics and Engineering doi:10.1016/j.cma.2016.07.009.

Bennett, T.D., Rothe, C.F., 1981. Hepatic capacitance responses to changes in flow and hepatic venous pressure in dogs. American Journal of Physiology-Heart and Circulatory Physiology 240, H18-H28.

Bezy-Wendling, J., Kretowski, M., Mescam, M., Jurczuk, K., Eliat, P.A., 2007. Simulation of hepatocellular carcinoma in mri by combined macrovascular and pharmacokinetic models, in: Biomedical Imaging: From Nano to Macro, 2007. ISBI 2007. 4th IEEE International Symposium on, IEEE. pp. 1272-1275. 
Blanco, P.J., Feijóo, R.A., 2013. A dimensionally-heterogeneous closed-loop model for the cardiovascular system and its applications. Med Eng Phys 35, 652-67. doi:10.1016/j.medengphy.2012.07.011.

Bonfiglio, A., Leungchavaphongse, K., Repetto, R., Siggers, J.H., 2010. Mathematical modeling of the circulation in the liver lobule. Journal of biomechanical engineering 132, 111011.

Childress, E.M., Kleinstreuer, C., 2014. Impact of fluid-structure interaction on direct tumortargeting in a representative hepatic artery system. Annals of biomedical engineering 42, 461-474.

Chu, T.M., Reddy, N.P., 1992. A lumped parameter mathematical model of the splanchnic circulation. Journal of biomechanical engineering 114, 222-226.

Court, F., Wemyss-Holden, S., Morrison, C., Teague, B., Laws, P., Kew, J., Dennison, A., Maddern, G., 2003. Segmental nature of the porcine liver and its potential as a model for experimental partial hepatectomy. British journal of surgery 90, 440-444.

Debbaut, C., De Wilde, D., Casteleyn, C., Cornillie, P., Van Loo, D., Van Hoorebeke, L., Monbaliu, D., Fan, Y.D., Segers, P., 2012. Modeling the impact of partial hepatectomy on the hepatic hemodynamics using a rat model. Biomedical Engineering, IEEE Transactions on 59, 3293-3303.

Debbaut, C., Monbaliu, D., Segers, P., 2014a. Validation and calibration of an electrical analog model of human liver perfusion based on hypothermic machine perfusion experiments. International Journal of Artificial Organs 37, 486-98.

Debbaut, C., Vierendeels, J., Siggers, J.H., Repetto, R., Monbaliu, D., Segers, P., 2014b. A 3d porous media liver lobule model: the importance of vascular septa and anisotropic permeability for homogeneous perfusion. Computer methods in biomechanics and biomedical engineering 17 , $1295-1310$.

Ho, H., Sorrell, K., Bartlett, A., Hunter, P., 2012. Blood flow simulation for the liver after a virtual right lobe hepatectomy, in: Medical Image Computing and Computer-Assisted InterventionMICCAI 2012. Springer, pp. 525-532. 
Iida, T., Yagi, S., Taniguchi, K., Hori, T., Uemoto, S., 2007. Improvement of morphological changes after $70 \%$ hepatectomy with portocaval shunt: preclinical study in porcine model. Journal of Surgical Research 143, 238-246.

LaDisa, J.F., Alberto Figueroa, C., Vignon-Clementel, I.E., Jin Kim, H., Xiao, N., Ellwein, L.M., Chan, F.P., Feinstein, J.A., Taylor, C.A., 2011. Computational Simulations for Aortic Coarctation: Representative Results From a Sampling of Patients. Journal of Biomechanical Engineering 133, 091008. doi:10.1115/1.4004996.

Lantz, B., Foerster, J., Link, D., Holcroft, J., 1981. Regional distribution of cardiac output: normal values in man determined by video dilution technique. American Journal of Roentgenology 137, 903-907.

Liang, F., Liu, H., 2005. A closed-loop lumped parameter computational model for human cardiovascular system. JSME International Journal 48, 484-493.

Liang, F., Takagi, S., Himeno, R., Liu, H., 2009. Multi-scale modeling of the human cardiovascular system with applications to aortic valvular and arterial stenoses. Medical \& biological engineering \& computing $47,743-755$.

Lukeš, V., Jiř́k, M., Jonášová, A., Rohan, E., Bublík, O., Cimrman, R., 2014. Numerical simulation of liver perfusion: from CT scans to FE model. arXiv preprint arXiv:1412.6412 .

Pedley, T.J., Brook, B.S., Seymour, R.S., 1996. Blood pressure and flow rate in the giraffe jugular vein. Philos Trans R Soc Lond B Biol Sci 351, 855-66. doi:10.1098/rstb.1996.0080.

Peeters, G., Debbaut, C., Cornillie, P., De Schryver, T., Monbaliu, D., Laleman, W., Segers, P., 2015. A multilevel modeling framework to study hepatic perfusion characteristics in case of liver cirrhosis. Journal of biomechanical engineering 137, 051007.

Pennati, G., Migliavacca, F., Dubini, G., Pietrabissa, R., de Leval, M.R., 1997. A mathematical model of circulation in the presence of the bidirectional cavopulmonary anastomosis in children with a univentricular heart. Medical Engineering \& Physics 19, 223-234. 
Pinzani, M., Vizzutti, F., 2005. Anatomy and vascular biology of the cells in the portal circulation, in: Portal hypertension. Springer. chapter 2, pp. 15-35.

R Development Core Team, 2008. R: A Language and Environment for Statistical Computing. R Foundation for Statistical Computing. Vienna, Austria. URL: http://www.R-project.org. ISBN 3-900051-07-0.

Raines, J.K., Jaffrin, M.Y., Shapiro, A.H., 1974. A computer simulation of arterial dynamics in the human leg. Journal of biomechanics 7, 77-91.

Ricken, T., Werner, D., Holzhütter, H., König, M., Dahmen, U., Dirsch, O., 2015. Modeling function-perfusion behavior in liver lobules including tissue, blood, glucose, lactate and glycogen by use of a coupled two-scale pde-ode approach. Biomechanics and modeling in mechanobiology $14,515-536$.

Robinson, A., 2016. Package 'equivalence'. Technical Report. https://cran.rproject.org/web/packages/equivalence/equivalence.pdf.

Robinson, A.P., Duursma, R.A., Marshall, J.D., 2005. A regression-based equivalence test for model validation: shifting the burden of proof. Tree physiology 25, 903-913.

Robinson, A.P., Froese, R.E., 2004. Model validation using equivalence tests. Ecological Modelling $176,349-358$.

Rypins, E.B., Rosenberg, K.M., Sarfeh, I.J., Houck, J., Conroy, R.M., Milne, N., 1987. Computer analysis of portal hemodynamics after small-diameter portacaval h-grafts: the theoretical basis for partial shunting. Journal of surgical research 42, 354-361.

Sainz-Barriga, M., Scudeller, L., Costa, M.G., de Hemptinne, B., Troisi, R.I., 2011. Lack of a correlation between portal vein flow and pressure: toward a shared interpretation of hemodynamic stress governing inflow modulation in liver transplantation. Liver Transplantation 17, 836-848.

Schwen, L.O., Schenk, A., Kreutz, C., Timmer, J., Rodríguez, M.M.B., Kuepfer, L., Preusser, T., 2015. Representative sinusoids for hepatic four-scale pharmacokinetics simulations. PloS one 10, e0133653. 
Segers, P., Stergiopulos, N., Schreuder, J.J., Westerhof, B.E., Westerhof, N., 2000. Left ventricular wall stress normalization in chronic pressure-overloaded heart: a mathematical model study. American Journal of Physiology-Heart and Circulatory Physiology 279, H1120-H1127.

Segers, P., Stergiopulos, N., Westerhof, N., Wouters, P., Kolh, P., Verdonck, P., 2003. Systemic and pulmonary hemodynamics assessed with a lumped-parameter heart-arterial interaction model. Journal of Engineering Mathematics 47, 185-199.

Serban, R., Petra, C., Hindmarsh, A., 2015. User documentation of ida v2.7.0 URL: https://computation.llnl.gov/casc/sundials/documentation/documentation.html.

Shapiro, A.H., 1977. Steady flow in collapsible tubes. Journal of Biomechanical Engineering 99, $126-147$.

Suga, H., Sagawa, K., 1974. Instantaneous pressure-volume relationships and their ratio in the excised, supported canine left ventricle. Circulation research 35, 117-126.

Swindle, M., Makin, A., Herron, A., Clubb, F., Frazier, K., 2012. Swine as models in biomedical research and toxicology testing. Veterinary Pathology Online 49, 344-356.

Van Der Plaats, A., t Hart, N., Verkerke, G., Leuvenink, H., Verdonck, P., Ploeg, R., Rakhorst, G., 2004. Numerical simulation of the hepatic circulation. The International journal of artificial organs $27,222-230$.

Vasavada, B.B., Chen, C.L., Zakaria, M., 2014. Portal flow is the main predictor of early graft dysfunction regardless of the grwr status in living donor liver transplantation-a retrospective analysis of 134 patients. International Journal of Surgery 12, 177-180.

Vignon-Clementel, I.E., Figueroa, C.A., Jansen, K.E., Taylor, C.A., 2010. Outflow boundary conditions for 3D simulations of non-periodic blood flow and pressure fields in deformable arteries. Computer Methods in Biomechanics and Biomedical Engineering 13, 625-640. URL: <Go to ISI > : //WOS : 000281851500012, doi:10.1080/10255840903413565. 


\begin{tabular}{|c|c|c|}
\hline & Average & Std \\
\hline \multicolumn{3}{|l|}{ Heart } \\
\hline $\mathrm{HR}(1 / \mathrm{min})$ & 90 & 8 \\
\hline$E a_{R A}\left(\mathrm{dyn} / \mathrm{cm}^{5}\right)$ & 153 & 23 \\
\hline$E b_{R A}\left(\operatorname{dyn} / \mathrm{cm}^{5}\right)$ & 55 & 42 \\
\hline$E a_{R V}\left(\mathrm{dyn} / \mathrm{cm}^{5}\right)$ & 798 & 9 \\
\hline$E b_{R V}\left(\mathrm{dyn} / \mathrm{cm}^{5}\right)$ & 66 & 42 \\
\hline$E a_{L A}\left(\mathrm{dyn} / \mathrm{cm}^{5}\right)$ & 200 & 0 \\
\hline$E b_{L A}\left(\mathrm{dyn} / \mathrm{cm}^{5}\right)$ & 400 & 0 \\
\hline$E a_{L V}\left(\mathrm{dyn} / \mathrm{cm}^{5}\right)$ & 1992 & 29 \\
\hline$E b_{L V}\left(\mathrm{dyn} / \mathrm{cm}^{5}\right)$ & 318 & 85 \\
\hline$V_{R A .0}(\mathrm{ml})$ & 4 & 0 \\
\hline$V_{R V .0}(\mathrm{ml})$ & 10 & 0 \\
\hline$V_{L A .0}(\mathrm{ml})$ & 4 & 0 \\
\hline$V_{L V .0}(\mathrm{ml})$ & 5 & 0 \\
\hline \multicolumn{3}{|l|}{ Other Organs } \\
\hline$R_{p}^{O O}\left(\right.$ dyn.s $\left./ \mathrm{cm}^{5}\right)$ & 95.8 & 26.7 \\
\hline$R_{d}^{O O}\left(\right.$ dyn.s $\left./ \mathrm{cm}^{5}\right)$ & 1809.1 & 1030.5 \\
\hline$C^{O O}\left(\mathrm{~cm}^{5} / \mathrm{dyn}\right)$ & $7.36 \mathrm{e}-4$ & 0 \\
\hline \multicolumn{3}{|l|}{ Digestive Organs } \\
\hline$R_{p}^{D O}\left(\right.$ dyn.s $\left./ \mathrm{cm}^{5}\right)$ & 478.3 & 111.9 \\
\hline$R_{d}^{D O}\left(\right.$ dyn.s $\left./ \mathrm{cm}^{5}\right)$ & 4305.8 & 1006.7 \\
\hline$C^{D O}\left(\mathrm{~cm}^{5} / \mathrm{dyn}\right)$ & $3 e-4$ & 0 \\
\hline \multicolumn{3}{|l|}{ Liver } \\
\hline$R_{h a}\left(\right.$ dyn.s $\left./ \mathrm{cm}^{5}\right)$ & $2.81 \mathrm{e} 4$ & $1.64 \mathrm{e} 4$ \\
\hline$R_{p v}\left(\right.$ dyn.s $\left./ \mathrm{cm}^{5}\right)$ & $3.71 \mathrm{e} 2$ & $3.10 \mathrm{e} 2$ \\
\hline$R_{l}\left(\right.$ dyn.s $\left./ \mathrm{cm}^{5}\right)$ & 7.36Ee1 & $5.83 \mathrm{e} 1$ \\
\hline M (g) & 863.2 & 88.3 \\
\hline$M_{l l}(\mathrm{~g})$ & 207.3 & 101.8 \\
\hline$M_{m l}(\mathrm{~g})$ & 417.0 & 74.7 \\
\hline$M_{r l}(\mathrm{~g})$ & 238.8 & 72.1 \\
\hline
\end{tabular}

Table 5: 0D closed-loop model parameter values average and standard deviation for the 12 animal simulations.

\section{Appendix}

In this appendix, tables present the closed-loop lumped parameter averages and standard deviations and the results of the twelve simulations with and without blood volume change. 


\begin{tabular}{|c|c|c|c|c|c|c|c|c|c|c|c|c|}
\hline Animals & iF01 & $\mathrm{iF} 02$ & iF03 & iF06 & iF08 & iF09 & iF010 & iF011 & iF012 & iF014 & iF016 & iF2A \\
\hline \multicolumn{13}{|l|}{$P_{a}(\mathrm{mmHg})$} \\
\hline pre & 63.5 & 50 & 48.7 & 49.7 & 35.5 & 60.5 & 54.2 & 51.8 & 58.6 & 45.2 & 76.7 & 52.7 \\
\hline post constant vol & 64.1 & 52.4 & 49.4 & 51.5 & 36.6 & 63.7 & 57.9 & 53.6 & 60.6 & 48 & 78.3 & 53.6 \\
\hline post vol change & 56 & 28.5 & 49.4 & 38.5 & 41.3 & 52.3 & 50.4 & 43.9 & 49.9 & 48 & 53.7 & 39.2 \\
\hline vol change (ml) & -200 & -700 & 0 & -400 & 200 & -300 & -200 & -300 & -300 & 0 & -500 & -400 \\
\hline \multicolumn{13}{|l|}{$P_{p v}(\mathrm{mmHg})$} \\
\hline pre & 6.6 & 5.5 & 7 & 8.4 & 6 & 8.1 & 9.7 & 10.6 & 10.9 & 4 & 5 & 4.1 \\
\hline post constant vol & 8.22 & 10 & 9.1 & 12.6 & 7.8 & 16.6 & 17.3 & 17.9 & 19.1 & 8.7 & 8.5 & 6.5 \\
\hline post vol change & 7.4 & 6.3 & 9.1 & 10 & 8.7 & 14.2 & 15.5 & 15.2 & 16.3 & 8.7 & 6.4 & 5.1 \\
\hline vol change (ml) & -200 & -700 & 0 & -400 & 200 & -300 & -200 & -300 & -300 & 0 & -500 & -400 \\
\hline \multicolumn{13}{|l|}{$P_{p v}-P_{v}(\mathrm{mmHg})$} \\
\hline pre & 2 & 2.3 & 1.3 & 3.1 & 1.3 & 7.4 & 7.6 & 9.2 & 9.2 & 2.2 & 3 & 2.8 \\
\hline post constant vol & 3.62 & 6.8 & 3.4 & 7.3 & 3.2 & 15.9 & 15.3 & 16.5 & 17.4 & 6.9 & 6.5 & 5.2 \\
\hline post vol change & 3.4 & 4.6 & 3.4 & 6.1 & 3.5 & 13.7 & 13.7 & 14 & 14.9 & 6.9 & 5 & 4.2 \\
\hline vol change (ml) & -200 & -700 & 0 & -400 & 200 & -300 & -200 & -300 & -300 & 0 & -500 & -400 \\
\hline \multicolumn{13}{|l|}{$Q_{h a}(\mathrm{~L} / \mathrm{min})$} \\
\hline pre & 0.12 & 0.22 & 0.06 & 0.2 & 0.2 & 0.21 & 0.24 & 0.18 & 0.21 & 0.23 & 0.11 & 0.11 \\
\hline post constant vol & 0.05 & 0.04 & 0.01 & 0.05 & 0.06 & 0.05 & 0.06 & 0.05 & 0.05 & 0.04 & 0.03 & 0.04 \\
\hline post vol change & 0.04 & 0.02 & 0.01 & 0.04 & 0.06 & 0.04 & 0.05 & 0.04 & 0.04 & 0.04 & 0.02 & 0.03 \\
\hline vol change (ml) & -200 & -700 & 0 & -400 & 200 & -300 & -200 & -300 & -300 & 0 & -500 & -400 \\
\hline \multicolumn{13}{|l|}{$Q_{p v}(\mathrm{~L} / \mathrm{min})$} \\
\hline pre & 0.98 & 1 & 0.59 & 1.12 & 0.73 & 0.68 & 0.88 & 0.64 & 0.69 & 0.67 & 0.97 & 0.67 \\
\hline post constant vol & 0.96 & 1 & 0.58 & 1.1 & 0.71 & 0.61 & 0.81 & 0.56 & 0.6 & 0.64 & 0.95 & 0.66 \\
\hline post vol change & 0.84 & 0.52 & 0.58 & 0.78 & 0.8 & 0.49 & 0.69 & 0.45 & 0.49 & 0.64 & 0.64 & 0.48 \\
\hline vol change (ml) & -200 & -700 & 0 & -400 & 200 & -300 & -200 & -300 & -300 & 0 & -500 & -400 \\
\hline
\end{tabular}

Table 6: Simulation results for the 12 different pigs before resection (pre), after resection with constant blood volume (post constant vol) and after resection with constant blood volume (post vol change) for : arterial pressure $P_{a}$, portal vein pressure $P_{p v}$, pressure drop $P_{p v}-P_{v}$, hepatic artery flow $Q_{h a}$ and portal vein flow $Q_{p v}$. The amount of volume added or removed for the simulation is given (vol change line). 